\title{
IMPROPER DOUBLE INTEGRALS
}

BY C. A. SHOOK

1. Introduction. It can be shown by examples that there exist integrals of the type

$$
\int_{a}^{c} d y \int_{b}^{d} f(x, y) d x
$$

where $a, b, c$ and $d$ are constants, in which an alteration of value occurs when the order of integration is reversed. Jordan* has given the following example. Let

$$
f(x, y)=\frac{\partial^{2} V}{\partial x \partial y},
$$

where $V=\arctan (y / x) ;$ and let $a=b=0$, and $c=d=1$. I adduce one more example in which

$$
f(x, y)=2 \frac{x-y}{(x+y)^{3}},
$$

and in which the limits are the same as in the previous example. In this example, the value obtained is -1 or +1 according as we integrate first with respect to $x$ or with respect to $y$.

Conditions sufficient to change the order of integration have been investigated by de la Vallee Poussin, $t$ who showed that the whole question rests on the uniform convergence of the improper integrals involved. The question has also been studied by Brunel, $\$$ Jordan,* Stolz, $\S$ and Osgood.

* Cours d'Analyse, vol. 2, pp. 66 et seq.

† Sur la convergence des intégrales définies, Journal DE Mathématiques, (4), vol. 8 (1892), p. 421.

† Encyklopädie der Mathematischen Wissenschaften, II, 1 , A, 1, p. 135 .

§ Differential- und Integralrechnung, vol. 3, Chapter 16.

II Annals of Mathematics, (2), vol. 3, p. 129. 
The following theorem is due to Osgood:

Let $f(x, y)$ be continuous in the region $a \leqq x, b \leqq y$, and let

(1) $\int_{b}^{\infty} f(x, y) d y$ converge uniformly in an arbitrary finite

(2) $\quad \int_{a}^{\infty} f(x, y) d x$ converge uniformly in an arbitrary finite interval, $a \leqq x \leqq G$

(3) $\quad \int_{a}^{\infty} d x \int_{b}^{y} f(x, y) d y$ converge uniformly in the infinite interval, $b \leqq y \leqq \infty$.

Then $\int_{a}^{\infty} d x \int_{b}^{\infty} f(x, y) d y$ and $\int_{b}^{\infty} d y \int_{a}^{\infty} f(x, y) d x$ both converge and are equal.

The purpose of this paper is to show that if we introduce the hypothesis that $f(x, y)$ does not change sign in the region, then (3) above can be replaced by the condition that

$$
\int_{a}^{\infty} d x \int_{b}^{\infty} f(x, y) d y \text { converge. }
$$

Next, the theorem as thus modified will be extended to include the case in which the integrand does not remain finite at either or both lower limits. Finally the extended theorem will be applied to an important iterated integral arising in the theory of the gamma function.

2. Definitions and Lemma. Let $f(x, y)$ be continuous, $c \leqq x \leqq d, a<y$. Suppose that $f(x, y)$ does not remain finite as $y$ tends to $a$. Finally, let $b>a$. Then the integral, $\int_{b}^{\infty} f(x, y) d y$, (or the integral $\int_{a}^{b} f(x, y) d x$ ), is said to converge uniformly in the interval $c \leqq x \leqq d$, if, corresponding to an arbitrarily small positive quantity $\varepsilon$, there exists a positive quantity $Y$, (or a positive quantity $\delta$ ) independent of $x$, such that for every value of $x$ in its interval, $\left|\int_{y_{1}}^{y_{2}} f(x, y) d y\right|<\varepsilon$, provided $y_{1}, y_{2} \geqq Y$, (or $a \leqq y_{1}, y_{2} \leqq a+\delta$ ). To establish uniform convergence we have recourse to the Vallée-Poussin* $\mu$-test. This may be stated as follows.

* Loc. cit. 
If there exists a finction $\mu(y)$ such that

(A) $|f(x, y)| \leqq \mu(y)$ for all values of $x$ and $y$ in the region considered;

(B) $\int_{b}^{\infty} \mu(y) d y\left(\right.$ or $\left.\int_{a}^{b} \mu(y) d y\right)$ converges;

then $\int_{b}^{\infty} f(x, y) d y\left(\right.$ or $\left.\int_{a}^{b} f(x, y) d y\right)$ converges uniformly,

$$
c \leqq x \leqq d .
$$

We can now state and prove the following lemma.

LEMMA. If

(a) $f(x, y)$ is continuous, $a \leqq x \leqq b, \quad c \leqq y$;

(b) $\quad \int_{c}^{\infty} f(x, y) d y$ converges uniformly, $a \leqq x \leqq b$;

then $\int_{a}^{b} d x \int_{c}^{\infty} f(x, y) d y=\int_{c}^{\infty} d y \int_{a}^{b} f(x, y) d x$.

Since the integral $\int_{c}^{\infty} f(x, y) d y$ converges uniformly, $a \leqq x \leqq b$, it represents a continuous function of $x$ in that interval, so that the integral

$$
\int_{a}^{b} d x \int_{c}^{\infty} f(x, y) d y
$$

has a definite meaning. Let $n$ be any number greater than $c$ and write

$$
\int_{a}^{b} d x \int_{c}^{\infty} f(x, y) d y--\int_{a}^{b} d x \int_{c}^{n} f(x, y) d y=\int_{a}^{b} d x \int_{n}^{\infty} f(x, y) d y .
$$

Also, the absolute value of the left hand member is equal to the absolute value of the right hand member. But, by (b), we have

$$
\left|\int_{n}^{\infty} f(x, y) d y\right|<\varepsilon, n>m, \quad m \text { independent of } x,
$$

where $\varepsilon$ is an arbitrarily small positive number and $m$ is a positive number corresponding to $\varepsilon$. Also

$$
\int_{a}^{\triangleright b} d x \int_{c}^{n} f(x, y) d y=\int_{c}^{n} d y \int_{a}^{b} f(x, y) d x
$$

since both are equal to a certain definite volume. Hence 
$\left|\int_{a}^{b} d x \int_{c}^{\infty} f(x, y) d y-\int_{c}^{n} d y \int_{a}^{b} f(x, y) d x\right|<\varepsilon(b-a), \quad n>m$, and this proves the lemma.

3. The Modified Theorem. In proving the modified theorem, use is made of an important theorem of Osgood* which may be stated as follows:

Let $F(x, y)$ be a function of the two independent variables $x$ and $y$, satisfying the following conditions:

(1) $\lim F(x, y)$ exists, say equals $f(y)$;

(2) $\lim _{x \rightarrow a} F(x, y)$ exists, say equals $g(x)$;

(3) $\stackrel{y \rightarrow b}{F}(x, y)$ converges uniformly when $x$ tends to a, that is, $\left|\boldsymbol{F}\left(x^{\prime}, y\right)-F\left(x^{\prime \prime}, y\right)\right|<\varepsilon, a \leqq x^{\prime}, x^{\prime \prime} \leqq a+\delta, \delta$ independent of $y$.

Then we conclude

(4) $\lim _{y \rightarrow b} f(y)$ exists, say equals $A$;

(5) $\lim _{x \rightarrow a} g(x)$ exists, say equals $B$;

(6) $A=B$.

The theorem, with the proper modification of the third condition, holds when $a$ and $b$ are infinite.

Suppose now that $f(x, y)$ remains always positive, and let

$$
F(x, y)=\int_{a}^{x} d x \int_{b}^{y} f(x, y) d y=\int_{b}^{y} d y \int_{a}^{x} f(x, y) d x .
$$

We will show that $F(x, y)$ satisfies the conditions of the theorem just stated, taking the $a$ and $b$ of the theorem infinite. We have

$$
\begin{gathered}
\lim _{x \rightarrow \infty} \int_{a}^{x} d x \int_{b}^{y} f(x, y) d y=\int_{a}^{\infty} d x \int_{b}^{y} f(x, y) d y \\
=\int_{b}^{y} d y \int_{a}^{\infty} f(x, y) d x, \text { by the Lemma } \\
\lim _{y \rightarrow \infty} \int_{b}^{y} d y \int_{a}^{x} f(x, y) d x=\int_{b}^{\infty} d y \int_{a}^{x} f(x, y) d x \\
=\int_{a}^{x} d x \int_{b}^{\infty} f(x, y) d y, \text { by the Lemma. }
\end{gathered}
$$

* Funktionentheorie, vol. 1, p. 519. This theorem is fundamental in the consideration of double limits, on which see Osgood, this Bulletin, rol. 3, p. 59; also Funktionentheorie, vol. 1, p. 66. 
We now have to show that $F(x, y)$ converges uniformly when $x$ increases without limit, that is, that

$$
\left|\int_{a}^{x^{\prime}} d x \int_{b}^{y} f(x, y) d y-\int_{a}^{x^{\prime \prime}} d x \int_{b}^{y} f(x, y) d y\right|<\varepsilon
$$

provided $x^{\prime}$ and $x^{\prime \prime}$ are both greater than a certain suitably chosen number. Combining integrals, we have

$$
\left|\int_{x^{\prime}}^{x^{\prime \prime}} d x \int_{b}^{y} f(x, y) d y\right|<\varepsilon
$$

This is precisely the condition that the integral

$$
\int_{a}^{\infty} d x \int_{b}^{y} f(x, y) d y
$$

should converge uniformly. To apply the $\mu$-test, take

$$
\mu(x)=\int_{b}^{\infty} f(x, y) d y
$$

and notice that the conditions of the test are satisfied. Hence

or

$$
\lim _{y \rightarrow \infty} \int_{b}^{y} d y \int_{a}^{\infty} f(x, y) d x=\lim _{x \rightarrow \infty} \int_{a}^{x} d x \int_{b}^{\infty} f(x, y) d y
$$

$$
\int_{b}^{\infty} d y \int_{a}^{\infty} f(x, y) d x=\int_{a}^{\infty} d x \int_{b}^{\infty} f(x, y) d y
$$

which proves the modified theorem.

4. The Extended Theorem. We may now state a more extended theorem.

Theorem A. Let $f(x, y)$ be a function of $x$ and $y$ satisfying the conditions

(1) $f(x, y)$ is continuous, $a<x, b<y$, and may increase without limit as $x$ tends to $a$ or as $y$ tends to $b$;

(2) $f(x, y) \geqq 0$ in the region considered;

(3) $\int_{a}^{c} f(x, y) d x$ converges uniformly, $b<t \leqq y, \quad c>a$;

(4) $\int_{c}^{\infty} f(x, y) d x$ converges uniformly, $b<t \leqq y, \quad c>a$;

(5) $\int_{b}^{d} f(x, y) d y$ converges uniformly, $a<s \leqq x, d>b$;

(6) $\int_{d}^{\infty} f(x, y) d y$ converges uniformly, $a<s \leqq x, \quad d>b$; 
(7) $\int_{a}^{\infty} d x \int_{b}^{\infty \infty} f(x, y) d y$ converges.

Then the order of integration can be reversed, that is,

$$
\int_{a}^{\infty} d x \int_{b}^{\infty} f(x, y) d y=\int_{b}^{\infty} d y \int_{a}^{\infty} f(x, y) d x
$$

To prove this theorem, we write, omitting the integrand for brevity,

$$
\int_{a}^{\infty} \int_{b}^{\infty}=\int_{a}^{c} \int_{b}^{d}+\int_{a}^{c} \int_{d}^{\infty}+\int_{c}^{\infty} \int_{b}^{d}+\int_{c}^{\infty} \int_{d}^{\infty} .
$$

The last of the four integrals of the right hand member falls directly under the modified theorem, stated in $\S 1$ and proved in $\S 3$. The other three integrals can be dealt with in a precisely similar way by making obvious changes. The hypotheses under Theorem A suffice for these proofs. But when the order of integration is changed in each of the four integrals, their sum becomes

$$
\int_{b}^{\infty} \int_{a}^{\infty}
$$

and this proves the theorem.

5. Application of Theorem A to a Particular Integral. In obtaining the relation between beta and gamma functions, *

$$
B(m, n)=\frac{\Gamma(m) \cdot \Gamma(n)}{\Gamma(m+n)}
$$

it is necessary to change the order of integration of the integral

$$
\int_{0}^{\infty} d y \int_{0}^{\infty} x^{m-1} y^{m+n-1} e^{-y(1+x)} d x
$$

where $m$ and $n$ are any positive numbers. This change of order can be justified by Theorem A. To apply the theorem take $c=d=1$. Conditions 1,2 and 7 are obviously satisfied. To show that conditions 3 to 6 inclusive

* Byerly, Integral Calculus, p. 113. 
are satisfied we have to exhibit a set of $\mu$-functions covering the various cases. Such a set follows:

\begin{tabular}{|c|c|c|}
\hline & $0<t \leqq y \leqq 1$ & $1 \leqq y$ \\
\hline $\int_{0}^{1} f(x, y) d x$ & $\begin{aligned} \mu(x)= & x^{m-1} e^{-t x} G \\
& 1<m+n \\
= & x^{m-1} e^{-t x} H, \\
& 0<m+n<1\end{aligned}$ & $\mu(x)=x^{m-1} e^{-x} G$ \\
\hline \multirow[t]{2}{*}{$\int_{1}^{\infty} f(x, y) d x$} & $\begin{aligned} \mu(x)= & x^{m-1} e^{-t x} G \\
& 1<m+n \\
= & x m-1 e^{-t x} H \\
& 0<m+n<1\end{aligned}$ & $\mu(x)=x^{m-1} e^{-x} G$ \\
\hline & $0<s \leqq x \leqq 1$ & $1 \leqq x$ \\
\hline $\int_{0}^{1} f(x, y) d y$ & $\begin{aligned} \mu(x)= & y^{n} e^{-y} M \\
& 1<m \\
= & y^{m+n-1} e^{-y} N \\
& 0<m \leqq 1\end{aligned}$ & $\begin{aligned} \mu(y)= & y^{n} e^{-y} M \\
& 1<m \\
= & y^{m+n-1} e^{-y} \\
& 0<m \leqq 1\end{aligned}$ \\
\hline $\int_{1}^{\infty} f(x, y) d y$ & $\begin{aligned} \mu(x)= & y^{m+n-1} e^{-y} M \\
& 1<m \\
= & y^{m+n-1} e^{-y} N \\
& 0<m \leqq 1\end{aligned}$ & $\mu(y)=y^{m+n-1} e^{-y} M$ \\
\hline$G=$ & $\left.\frac{m+n-1 \mid}{\rho}\right|^{|n-1| m+}$, & $H=e^{-t} t^{m+n-1}$ \\
\hline$M=$ & $m-1||^{|m-1|}$ & $N=s^{m-1}$ \\
\hline
\end{tabular}

$f(x, y)$ denotes the integrand of the integral under consideration.

YALE UnIVERsity 